\title{
The Design of Network Testing System for College Physics Experiment
}

\author{
Guo-Liang Fan*, Jun-Jie Liu, Ri Na \\ Department of Physics, School of Physical Science and Technology, Inner Mongolia University, Hohhot, China \\ (eeguoliangfan@sina.com)
}

\begin{abstract}
This paper introduced a system for college physics experiment testing by using the soft and hard resources and the opening technology of WEB. The rule of constructing the test paper is randomly choosing according to the difficulty coefficient, and the rule can improve the confidentiality, objectivity and fairness of the paper. At the same time, the system has the function of questions editing, constructing the test paper, online testing, score management, etc. The system can improve the working efficiency and teaching effect of college physics experiment and reduce the workload of teachers. It gives a valuable reference for the testing of other public courses.
\end{abstract}

Keywords —online testing, college physics experiment, ASP, educational administration management

\section{大学物理实验网络考试系统设计 \\ 校国梁 刘俊杰 那日 \\ 内蒙古大学物理科学与技术学院, 内蒙古 呼和浩特}

摘 要 文章介绍了一种利用已有软件、硬件资源和开放的 web 技术设计的大学物理实验考试系统。系统采用根据难度系数随机 组卷的原则, 增强了试卷的保密性、客观性和公平性。同时, 系统还具有试题编辑、随机组卷、在线测试、成绩管理等功能, 提高了 大学物理实验教学的工作效率和教学效果, 减轻了老师的工作量, 为其它公共课程的考试提供了一种有价值的参考。

关键词 精品课程, 教学改革, 大学物理实验

1. 引言

随着计算机的普及, 考试网络建设越来越成熟, 网络 在线考试系统在考试中的应用越来越多。大学物理实验是 高校各工科专业的必修课程之一, 而教务管理工作所涉及 的命题、组卷、印刷、组织考试、阅卷等工作量非常大, 以及传统笔试试卷不利于试卷标准化, 有必要将该课程的 部分考试内容借助于网络实验室, 采用无纸化网络考试。

随着时代的发展, 计算机技术迅速发展, 科技进步对 于教育提出的要求越来越高, 机试逐步代替笔试成为很多 课程的考试方式。所以对于大学物理实验课后考试、考查 也将逐步进入机试考试的大潮中。所以本文旨在于设计一 套基于 ASP 技术的大学物理实验考试系统 ${ }^{[1,2]}$, 用于解决实 验考试中工作量大、工作效率低下的问题, 为实践课程的

内蒙古自治区精品课程 “大学物理实验” 项目支持。
教学管理网络化注入新的元素。

\section{2. 系统模块结构划分}

对于网络考试来说, 针对的对象分为学生、教师和管 理员，每个角色都有不同的权限和任务。因此，在设计系 统时, 要充分考虑每个对象之间的相互关系, 以对象任务 为主体进行模块划分, 分为管理员模块、教师模块和学生 模块 ${ }^{[3,4]}$ 。三个模块的功能关系如图 1 所示。

\section{1 管理员模块}

该模块主要完成试题库的维护, 包括试题的查询、录 入、修改、组卷等功能。对试题的直接查找难度较大, 我 们采用模糊查询方式, 确保查到需求的试题。先查到试题 的题号, 并调出该题目的相关内容, 然后对该试题进行修 改。另外, 通过查询功能在组卷时可以方便快捷地找到所 


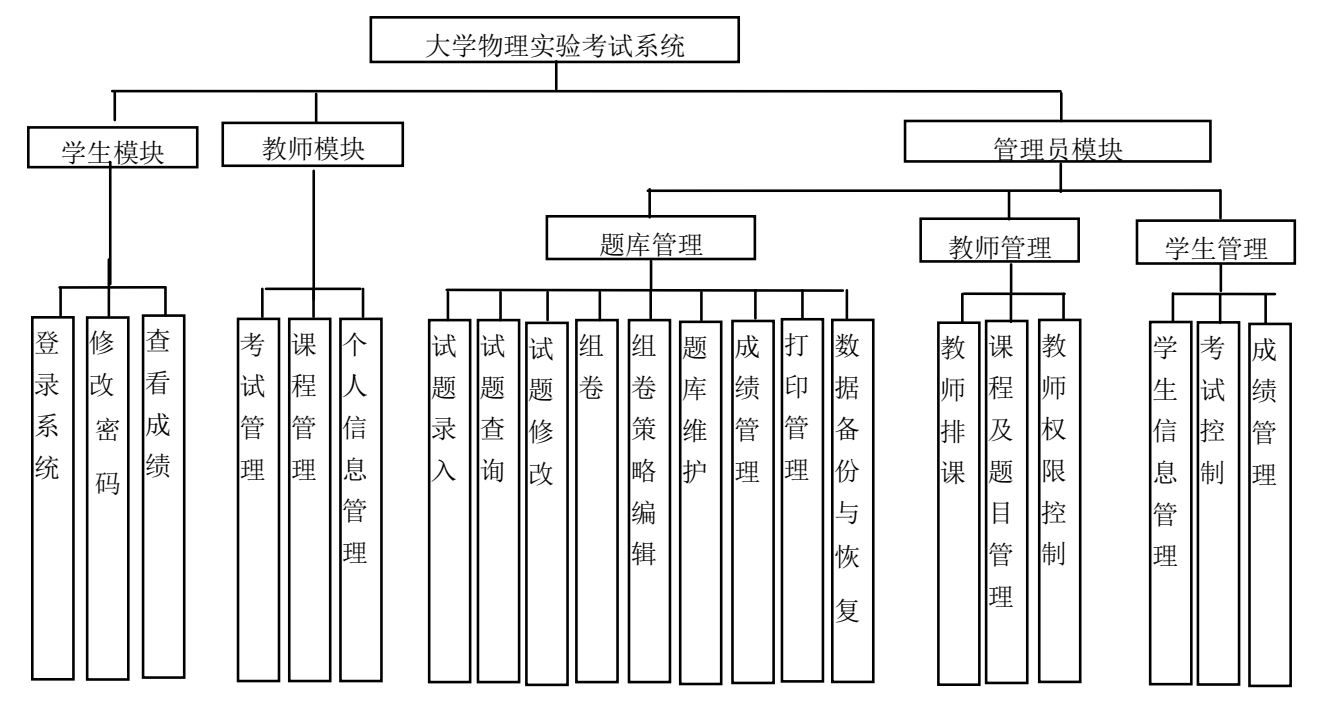

图 1 系统功能框图

需的试题。试题录入功能是对题库中试题的更新, 录入时 除了试题需要的题面、答案以外, 还要录入一些其他信息, 比如试题类型、难度系数、适合专业等信息, 以方便组卷 时控制组卷策略。组卷是考试系统设计的核心, 我们设计 了两种考试模式: 统一考试和每个实验考一个题。如果按 照第一种模式, 管理员可以选择一定的组卷策略, 通过控 制参数的输入, 自动生成试卷。按照学生的学号、实验时 间进行考试题目的选择。在系统数据库设计中利用一个抽 题规则库, 通过设定不同难度的试题及不同题目类型的出 题数量以确定考试内容; 然后, 根据所需要的试题量产生 随机数, 进行抽题; 最后, 将所抽出的题放入试卷库, 则 生成试卷。对不同的学生会重复这个生成试题的过程。如 果是按照一个实验一个题目的原则进行考试

管理员模块除了对试题进行控制外, 还要对学生进行 控制, 包括对学生信息的确认, 判断该学生是否在某一时 段进行了物理实验并判断该学生是否可以进行考试。

管理员模块还包括对大学物理实验的老师进行管理, 即判断教师负责的实验以及上课时间。

\section{2 教师模块}

教师模块相对简单, 登录后点击 “考试管理” 按钮, 学生输入学号即进入考试状态。由于开放式实验教学, 学 生可能在同一天进行了两个实验题目, 所以, 按照第二种 考试模式, 就需要根据学生当天实验情况作出判断, 学生 是参加哪个实验相对应的考试, 给出正确的题面。

\section{3 学生模块}

学生模块的作用是为了使学生可以及时、清晰的得知
自己对于考试内容模块是否掌握, 可以方便的得知自己的 成绩。

\section{3. 考试流程}

当系统进行考试时, 必须按照一定的流程进行, 如图 2 所示, 当学生输入学号, 然后根据学号、老师姓名以及 时间进行判断, 首先确定该生是否有资格考试, 以及是否 过了考试时间或未到考试时间进行处理, 最后确定该次实 验题目, 根据实验题目进行抽题考试。

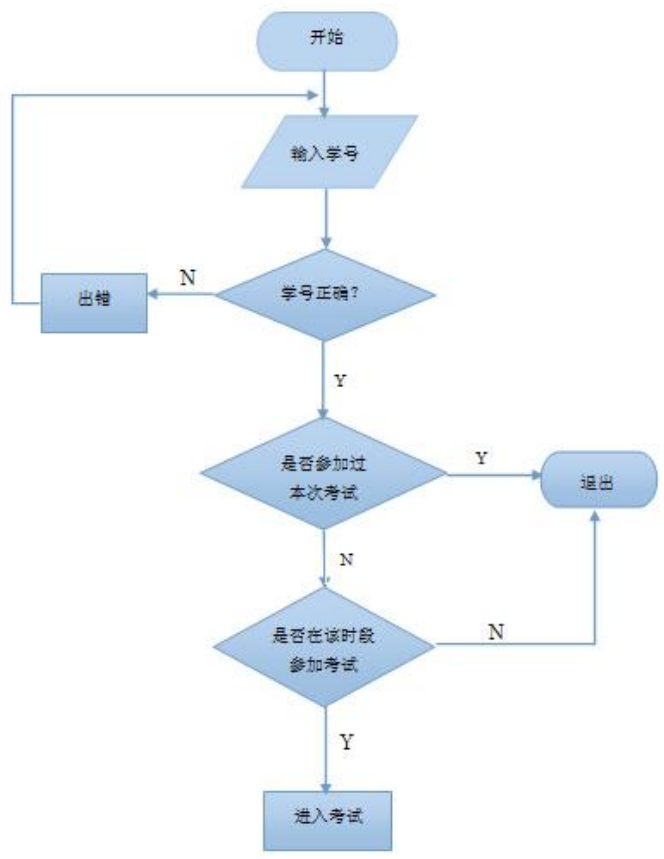

图 2 考试流程图 
考试过程中有考试时间提醒, 考生在完成答题后需要 进行交卷操作, 或超过考试时间系统将自动进行交卷操作。

当系统进行统一考试模式处理时, 就不需要以上判 断, 只要管理员组好试卷, 选好考试班级, 学生输入学 号即可从组好的试卷中抽取一份试卷进行答题, 和每个 实验考一个题不同的是考试的题目数量有所区别。

\section{3. 结束语}

在大学物理实验考试系统的实际使用过程中对本程序 进行了必要的测试和修改, 不断进行完善, 而且对该系统 的数据库进行不断的添加、修改, 该实验考试系统基本趋 于完整和成熟, 在实验考试中扮演了非常重要的角色, 为 实验管理系统增加了更为丰富的功能, 节省了人力物力,
在实验考试中开创了新的方式, 同时为其它课程的考试提 供了更为灵活的模式。

\section{参考文献(References)}

[1] D. H. Zhang, ASP program and application. BeiJing: China Machine PRESS, 2002.

[2] C. Jeff, P. M. John, C\# All-In-One Desk Reference Dummies, Wiley publishing Inc, 2009.

[3] T. Zhang, "The Research and development of online test system," Information Science, vol. 21, no. 8, pp. 871-876, 2003.

[4] X. D. Cheng, "The Development and Implementation of online test system," Health Vocational Education, vol. 25, no. 7, pp.67-68, 2007. 